\title{
Stretched Exponential Relaxation and Independent Relaxation Modes
}

\author{
R.M.C. de Almeida ${ }^{1}$, N. Lemke ${ }^{2}$, and I.A. Campbell ${ }^{3,4}$ \\ ${ }^{1}$ Instituto de Física, Universidade Federal do Rio Grande do Sul, \\ Caixa Postal 15051 - 91501-970, Porto Alegre, RS, Brazil \\ ${ }^{2}$ Centro de Ciências Exatas e da Terra - Unisinos \\ Av. Unisinos, 950 93022-000 - São Leopoldo - RS - Brazil \\ ${ }^{3}$ Laboratoire des Verres, Université de Montpellier II \\ ++34095 Montpellier Cedex 5, France \\ ${ }^{4}$ Laboratoire de Physique des Solides, Université Paris Sud, 91405 Orsay, France
}

Received on 15 October, 2000

\begin{abstract}
We discuss the origin of stretched exponential relaxation in disordered Ising spin systems by writing the master equation on the phase space, and the evolution of local and global spin autocorrelation functions, in terms of independent relaxation modes, which are eigenvectors of the time evolution operator. In this sense it is shown that when the relaxation modes are spatially delocalized, both local and global autocorrelation functions may present non-exponential relaxation. We also analyze results for random walks on the dilute hypercube, which may be associated with the phase space of a disordered Ising spin system. As expected, the results show a stretched exponential relaxation near the percolation transition, since it deals with random walks on a fractal percolating cluster defined on a closed surface. We argue that the same type of topology is present in the available region of configuration space in Ising spin-glass systems near the glass transition, since these systems present very similar relaxation patterns in this temperature range.
\end{abstract}

\section{Introduction}

Slow relaxation near a phase transition presents many interesting features that reflect the nature of the transition. This phenomenon may be observed in many different kinds of materials, ranging from the original observations by Kohlrausch for the decay of residual charge on a Leiden jar [1], to such theoretical models as the trap model $[2,3]$, or random walkers on dilute hypercubes of high dimensions [4-8], and spin glasses above, or ferromagnets below their transition temperatures, structural glasses, etc. [For a recent review, see Ref. [9]].

There is a controversy that begins with the form of the relaxation, and intensifies when the discussion turns to the possible origins for this behavior. Two different forms of non-exponential relaxation of a memory function $q(t)$ are often found in the literature, originating either in theoretical models or invoked in analysis of experimental data. The first is

$$
q(t) \sim \exp \left[-c(\ln t)^{\frac{d}{(d-1)}}\right]
$$

as proposed by Randeria et al. [10] for short-range Ising spin-glasses with local spin dynamics above the spinglass transition temperature, but this asymptotic form has never been observed [11]. The point is that systems should relax for very long times to reach the asymptotic regime, rendering difficult a conclusive statement based on numerical simulations.

The second form of non-exponential relaxation of memory functions is typically given by a stretched exponential of the form

$$
q(t) \sim C \exp \left[-(t / \tau)^{\beta}\right]
$$

where $\tau$ is a relaxation time that diverges as the transition is approached and $\beta<1(\beta=1$ is the case of a pure exponential relaxation). This expression has been found consistent with many experiments on disordered systems [9] and numerical simulations of different model systems $[2-8,12]$.

In this paper we address this controversy by considering the master equation representing the evolution of a spin glass in phase space. In this manner, we can write the evolving density function in terms of the relaxation modes related to the transition matrix eigenvectors. The transition matrix contains information about the dynamics of the system, giving the rate for the system to pass from one configuration to another. In section II, we write the master equation for a general Ising 
spin system and discuss some of the previous theoretical and numerical results from this point of view. In section III, we write the master equation for random walkers on a decimated hypercube, and use this model as an analogy for the phase space a spin glass. In this way the glass transition is associated with a percolation transition and the slow relaxation is explained as the expected slowing down of dispersion of random walkers on percolation clusters embedded on closed surfaces. The results are presented and discussed in section IV and finally on section $\mathrm{V}$ we conclude.

\section{The master equation and re- laxation modes}

Two models have been proposed that may yield stretched exponential relaxation in the appropriate limits. The trap model [2] considers random walkers in a d-dimensional space where traps are randomly distributed. The memory function here is given by the number of living walkers after a given time. The expected lifetime of the walkers, that is, the average time it takes for a walker to meet a trap, is determined by the initial distance to the nearest trap. Consequently the distribution of relaxation times contributing to the memory function depends on the distribution of linear sizes of trap-free regions. Also, each walker is relaxing in a given region, with a defined relaxation time, that is, each walker is in an independent relaxing mode of the dynamics of the system. The result of averaging over these independent relaxation modes gives a stretched exponential due to the particular form of the relaxation-time distribution originating in the random localization of traps.

Another theoretical model yielding stretched exponential relaxation is the hierarchical model [3]. In this model the spins are organized in different hierarchical levels, each level containing a given number of spins. The spins in a given level are allowed to change states only after some condition has been satisfied by the spins at the level immediately below. As a consequence, the relaxation times are always increasing for increasing levels, since superior levels spins must wait for the spins at lower levels to relax before they can start relaxing. Each spin level then can be viewed as an independent mode with a defined relaxation time. The memory function is the spin autocorrelation function and each spin relaxation is essentially ruled by only one independent mode. The authors of this model propose a specific constraint on the evolution of upper levels yields stretched exponential relaxation for the global memory function.

The key issue in both models presented above is the fact that the (global) memory function is an average over local memory functions that relax exponentially with different relaxation times. In other words, the lo- cal memory functions are measuring the evolution of independent relaxation modes of the system. We can verify this statement by considering a system with $N$ Ising spins, in which the monitored global memory function is given as

$$
q(t)=\frac{1}{N} \sum_{i=1}^{N} S_{i}(t) S_{i}(0)
$$

and then writing a master equation, defined over the phase space of the system as

$$
\frac{\partial \rho(\vec{S}, t)}{\partial t}=\sum_{\left\{\overrightarrow{S^{\prime}}\right\}}\left[Q\left(\vec{S}, \overrightarrow{S^{\prime}}\right) \rho\left(\overrightarrow{S^{\prime}}, t\right)-Q\left(\overrightarrow{S^{\prime}}, \vec{S}\right) \rho(\vec{S}, t)\right]
$$

where $Q\left(\overrightarrow{S^{\prime}}, \vec{S}\right)$ is the transition rate from configuration $\vec{S}$ to configuration $\overrightarrow{S^{\prime}}$ and $\rho(\vec{S}, t)$ is the probability of finding the system in the configuration $\vec{S}$ at time t. The transition matrix contains information about the dynamics of the system and is different for different models. It is convenient to express the dynamics in a Dirac-like notation. Writing the above equation for discrete time, the state of the system can be formally obtained as

$$
|\rho(t)\rangle=\mathrm{e}^{-\Lambda t}|\rho(t=0)\rangle
$$

where $|\rho(t)\rangle$ represents the probability of finding the system in each possible configuration at time $t$, and $\Lambda$ is given by

$$
\Lambda\left(\vec{S}, \overrightarrow{S^{\prime}}\right)=Q\left(\vec{S}, \overrightarrow{S^{\prime}}\right)-\delta\left(\vec{S}-\overrightarrow{S^{\prime}}\right) \sum_{\left\{\overrightarrow{S^{*}}\right\}} Q\left(\overrightarrow{S^{*}}, \vec{S}\right)
$$

The eigenvalue equation of the transition matrix $\Lambda$ is

$$
\Lambda\left|\lambda_{k}\right\rangle=\lambda_{k}\left|\lambda_{k}\right\rangle
$$

where $k=1,2, \ldots, 2^{N}$ and the eigenvectors $\left\{\left|\lambda_{k}\right\rangle\right\}$ form a basis for the space of the solutions of the evolution equation. One can also build another basis, considering the states $|\vec{S}\rangle$ that represent a state where we have probability one of finding the system in $\vec{S}=\left(S_{1}, S_{2}, \ldots, S_{N}\right)$ and zero in any other configuration. In this case,

$$
\left\langle\vec{S} \mid \overrightarrow{S_{0}}\right\rangle=\delta\left(\vec{S}-\overrightarrow{S_{0}}\right)
$$

and $\rho(\vec{S}, t)=\langle\vec{S} \mid \rho(\vec{t})\rangle$. The stationary solution(s) of Eq.(4) are the eigenstate(s) of Eq.(7) with eigenvalue zero, normalized such that

$$
\sum_{\{\vec{S}\}}\langle\vec{S} \mid \lambda=0\rangle=1
$$

while for all other eigenstates, with $\operatorname{Re}(\lambda)<0$, the above sum gives zero. It means that some of the components $\langle\vec{S} \mid \lambda \neq 0\rangle$ are less than zero. This has two main implications: (i) all physical configurations may be represented by a linear combination of the eigenstates of 
the evolution operator provided it contains a stationary component such that all components $\langle\vec{S} \mid \rho(\vec{t})\rangle$ of the physical state are non-negative and (ii) the role played by these non stationary components, in relation to the stationary states, is to add probability to some configurations while subtracting probability from other configurations, conserving total probability. These two features are valid due to the probability conservation guaranteed by the evolution equation. The state of the system at time $t$ may then be written as

$$
|\rho(t)\rangle=\sum_{k=1}^{2^{N}} \mathrm{e}^{\lambda_{k} t}\left|\lambda_{k}\right\rangle\left\langle\lambda_{k} \mid \rho(t=0)\right\rangle
$$

where it becomes clear that the eigenvalues of the transition matrix give the relaxation modes of the system. Letting $\lambda_{k}=-1 / \tau_{k}$, the above equation becomes

$$
|\rho(t)\rangle=\sum_{k=1}^{2^{N}} e^{-t / \tau_{k}}\left|\tau_{k}\right\rangle\left\langle\tau_{k} \mid \rho(t=0)\right\rangle .
$$

Now, Eq.(11) gives the relaxation of the system as a whole in terms of its independent modes. The question now is to translate this relaxation of the probability density of the system defined over its phase space into the relaxation of the spin autocorrelation function that is the commonly used memory function for Ising spin systems. For that we first take

$$
|\rho(t=0)\rangle=\left|\overrightarrow{S^{0}}\right\rangle
$$

where $\overrightarrow{S^{0}}$ is the initial configuration of the system. We then take the product of Eq.(11) with $\langle\vec{S}|$, multiply by $S_{i}$ and $S_{i}^{0}$, that are the values of the $i$-th spin in configurations $\vec{S}$ and $\overrightarrow{S^{0}}$, respectively, and average over all configurations, to obtain the local spin autocorrelation function:

$$
q_{i}(t)=\sum_{k=1}^{2^{N}} e^{-t / \tau_{k}} f_{i}\left(\tau_{k}, \overrightarrow{S^{0}}\right)
$$

with

$$
f_{i}\left(\tau_{k}, \overrightarrow{S^{0}}\right)=\sum_{\{\vec{S}\}} S_{i} S_{i}^{0}\left\langle\vec{S} \mid \tau_{k}\right\rangle\left\langle\tau_{k} \mid \overrightarrow{S^{0}}\right\rangle
$$

Finally, the global spin autocorrelation function may be written as

$$
q(t)=\sum_{k=1}^{2^{N}} e^{-t / \tau_{k}} g\left(\tau_{k}, \overrightarrow{S^{0}}\right)
$$

with

$$
\begin{aligned}
g\left(\tau_{k}, \overrightarrow{S^{0}}\right) & =\sum_{\{\vec{S}\}}\left(\frac{1}{N} \sum_{i=1}^{N} S_{i} S_{i}^{0}\right)\left\langle\vec{S} \mid \tau_{k}\right\rangle\left\langle\tau_{k} \mid \overrightarrow{S^{0}}\right\rangle \\
& =\frac{1}{N} \sum_{i=1}^{N} f_{i}\left(\tau_{k}, \overrightarrow{S^{0}}\right)
\end{aligned}
$$

Both equations present a stationary part, corresponding to $\tau_{i}=\infty\left(\lambda_{i}=0\right)$ and a relaxation part related to smaller relaxation times $\left(\lambda_{i}<0\right)$.

Now, considering the relaxing component for the hierarchical model, which is a spin system so that the above memory function applies, we observe that essentially each spin relaxes according to its level independent mode, that is, the sum over different relaxation modes $k$ splits into sums for different levels. Consequently, each spin is expected to relax exponentially, but the sum over individual spin relaxation functions may yield a stretched exponential form for suitable constraints.

In more general spin systems, it has been argued that some unfrustrated spin clusters may form, such that the relaxation within a given cluster is exponential, with relaxation times defined by the cluster size. The sum of these different modes of exponential relaxation with an appropriate cluster size distribution could yield a global stretched exponential behavior. These clusters would be related to the Griffiths singularities[13], and hence spatial inhomogeneities would be responsible for the observed slow relaxation. However, this model implies that each spin should relax exponentially (each cluster corresponding to an independent relaxation mode), and spatial correlation between local spin relaxation modes would signal the appearance of unfrustrated clusters. Glotzer et al.[12] monitored the relaxation of two and three dimensional Ising spin glasses and found stretched exponential relaxation for individual spins, that is, the sum in the expression for the local autocorrelation function $q_{i}(t)$ in Eq.(13) also yields a stretched exponential, implying that various relaxation modes contribute to the relaxation of individual spins. In fact, it is the structure of $g\left(\tau_{k}, \overrightarrow{S^{0}}\right)$ and $f_{i}\left(\tau_{k}, \overrightarrow{S^{0}}\right)$ that governs the contribution of each relaxation mode for the global and local spin correlation functions, defining their overall relaxation behavior. For example, if the relaxation modes are not spatially localized, $q(t)$ could in principle present stretched exponential relaxation with a given $\tau$ and $\beta$, and so could the local relaxation functions with different values for these parameters. Different values of $\tau$ and $\beta$ have indeed been found Glotzer et al. [12].

\section{Ising spin systems and the hypercube}

From Eq.(4) and the evolution equations for the spin autocorrelation function, it becomes clear that it is interesting to investigate the origins of non-exponential relaxation by focusing on the phase space of the system. In fact, the phase space of a system with $N$ Ising spins is the set of the vertices of a hypercube of $N$ dimensions. Now, adding to this the fact that random walkers 
on percolation clusters embedded on flat spaces yield a sublinear diffusion equation $\left\langle r^{2}\right\rangle \sim t^{\beta}$ [14], while random walkers on closed surfaces such as a hypersphere give a pure exponential decay of the average autocorrelation function, $\langle\cos (\theta(t))\rangle \sim \exp (-t / \tau)$, one is led to the conjecture proposed by Campbell [4] that dispersion of random walkers on percolation clusters embedded in a closed surface such as the hypercube should follow $\left\langle r^{2}\right\rangle \sim \exp \left[-(t / \tau)^{\beta}\right]$, with $\beta \rightarrow 1 / 3$ and $\tau \rightarrow \infty$ as the percolation threshold is approached.

In recent simulations of a percolation cluster embedded in the surface of a hypersphere, Jund, Jullien and Campbell [15] show that stretched exponential relaxation is directly connected to the fractal nature of these clusters. Campbell's conjecture goes further, by considering the implications of these results to the relaxation of such disordered systems as, for example, Ising spin glasses. The idea is to regard the relaxation of an Ising spin glass coupled to a heat bath as a random walk through the available states in phase space. This highdimensional, closed space has its landscape changed as the temperature approaches the transition from above, since the number of available states decreases in this process.

Near the transition it is conjectured that the set of available states forms a percolating cluster, and that right at the transition point the giant cluster breaks into many finite clusters, representing the many different free energy valleys typical of spin glasses below the glass transition temperature. As temperature is lowered towards the transition, the topology of the cluster becomes more labyrinthine, slowing down the relaxation towards equilibrium. Observe that this picture considers the relaxation of a density function on the hypercube where each site is a possible configuration of the system. As the different vertices change their occupation density, the local and global spin autocorrelation functions change accordingly, and it may be possible that they are simultaneously influenced by many different relaxation modes.

The simplest case of the above scenario has been considered by Campbell and collaborators [4-7] through Monte Carlo simulations of a random walker on an $N$ dimensional hypercube in which some of the vertices have been deleted or prohibited to the walker. There is a critical fraction $p_{c}$ of allowed or occupied vertices below which no percolation cluster is formed. The simulations, which were performed for occupation fractions $p \geq p_{c}$, monitored the average Hamming distance $q(t)$ between the current and initial positions of the walker as a function of time, which can be associated with the global spin autocorrelation function. The results are compatible with Campbell's conjecture, but the noise at small values of $q(t)$ for long times did not allow a decisive conclusion. An alternative procedure is to consider the evolution of the probability density of finding a walker at given vertex by directly iterating the mas- ter equation of the system [8], which has yielded very precise results.

Start by imagining a hypercube in (high) dimension $N$ with a fraction $p$ of its $2^{N}$ vertices occupied at random. The critical percolation concentration is given by

$$
p_{c}=\kappa+\frac{3}{2} \kappa^{2}+\frac{15}{14} \kappa^{3}+\ldots
$$

where $\kappa=1 /(N-1)$. For $p>p_{c}$ there exists a giant spanning cluster made up of occupied sites having one or more occupied sites as neighbors [16]. For $p<p_{c}$ there are only small clusters (with less than $N$ elements).

Considering each configuration $\vec{S}$ as the binary representation of an integer $\sigma$, the density function $\rho(\vec{S}, t)$ becomes then a function of two integer variables, $\rho(\sigma, t)$, and the direct iteration of the master equation may easily be implemented on a computer. Considering values of $p$ above $p_{c}$, the system starts at a given vertex $\sigma_{0}$ belonging to the infinite cluster. The probability that the random walker jumps to a neighboring site is $1 / N$ if it is 'occupied' and zero otherwise, in which case the walker stays where it is. The discrete-time master equation then reads

$$
\begin{gathered}
\rho(\sigma, t+1)=\rho(\sigma, t)+ \\
\sum_{\left\{\sigma^{\prime}\right\}}\left[W\left(\sigma, \sigma^{\prime}\right) \rho\left(\sigma^{\prime}, t\right)-W\left(\sigma^{\prime}, \sigma\right) \rho(\sigma, t)\right]
\end{gathered}
$$

with transition matrix $W\left(\sigma^{\prime}, \sigma\right)$ conveniently redefined to describe the jumping probabilities during discrete time intervals.

As the iteration procedure evolves we monitor the memory function of the system, the spin auto correlation function, calculated as

$$
q(t)=\frac{1}{N} \sum_{i=1}^{N} \sum_{\{\sigma\}} \rho(\sigma, t) S_{i}^{0} S_{i}
$$

where $S_{i}^{0}$ and $S_{i}$ are the $i$-th spins of the configurations associated, respectively, with $\sigma_{0}$ and $\sigma$, that is

$$
\begin{aligned}
S_{i}^{0} & =\frac{\left(2^{i-1} \mathrm{AND} \sigma_{0}\right)}{2^{i-2}}-1 \\
S_{i} & =\frac{\left(2^{i-1} \mathrm{AND} \sigma\right)}{2^{i-2}}-1 .
\end{aligned}
$$

The advantage of this procedure is that for each transition matrix, representing one realization of a hypercube with occupation probability $p$, successive iterations of the master equation provide the average over all possible random walks starting from the same initial condition. Averages over different samples are still required, but the accuracy is significantly enhanced in comparison to Monte Carlo calculations.

Very near $p_{c}$ the direct iteration of the master equation becomes too slow and a second method may be 
applied. In this case, we take advantage of the reduced number of sites in the percolating cluster and diagonalize the evolution operator, obtain the eigenvalues and eigenvectors and use them directly in Eq.(15).

As the occupation probability $p$ decreases from 1.0 down to the percolation threshold, the occupied vertices develop a fractal percolation cluster: the vector state $\rho \overrightarrow{(t)}$ diffuses on a fractal aggregate. In this case, we may expect anomalous diffusion. We therefore study the relaxation of the autocorrelation for different values of $p$, above the percolation threshold.

\section{Results}

For each $p$ studied, we considered 100 realizations of the hypercube. Since we explicitly solve the master equation for this system we obtain exact results (to within numerical rounding errors) for the average over all possible random walks for each combination of a realization of the hypercube and one given starting point. Therefore by studying even a relatively small number of samples we can obtain very good estimates for the average relaxation function $q(t)$. The main problem with this approach is that even with the optimization used, cpu time and memory restricted us to "low" dimensions $N$, since the number of vertices in the phase space increases exponentially with $N\left(\sim 2^{N}\right)$. For convenience we chose to carry out explicit calculations in dimension $N=16.100$ samples were studied for each value of $p: p=0.073,0.10,0.24,0.36$ and 0.5 , while 500 samples were studied for $p=0.12$. For $p=0.073$, very near the percolation threshold for $N=16$, we diagonalized the evolution operator, while for the other values the dynamical equation was directly iterated.

Before discussing the results, it is interesting to point out that we expect three relaxation regimes: $i)$ At short times $q(t)$ will behave as $1-\alpha t$ where $\alpha$ is the average probability per unit time that a step will be taken, i.e., it is proportional to the fraction of occupied neighbors of a site on the giant cluster. This is the leading term in the exponential function $\exp (-(\alpha t))$, so at very short times the relaxation will always be exponential. ii). At longer times, as the system explores the labyrinthine geometry of the giant cluster, a slow relaxation regime appears. iii) Finally, at even longer times, finite-size effects become measurable and a crossover back to an exponential relaxation is expected to set in. This last crossover time has been shown to depend on sample size for systems where relaxation arises from two competing exponential processes [17].

The results for $q(t)$ are presented in two different ways to verify whether the relaxation is following a stretched exponential form and then obtain the fitting parameters. Fig. 1 shows the data obtained for $q(t)$ in a log-log plot for different values of $p$, together with the stretched exponential fits with $\beta(p)$ and $\tau(p)$ as given in Fig. 2. The normalization parameter $C$ is always close to 1 . It can be seen immediately that the fits are of excellent quality.

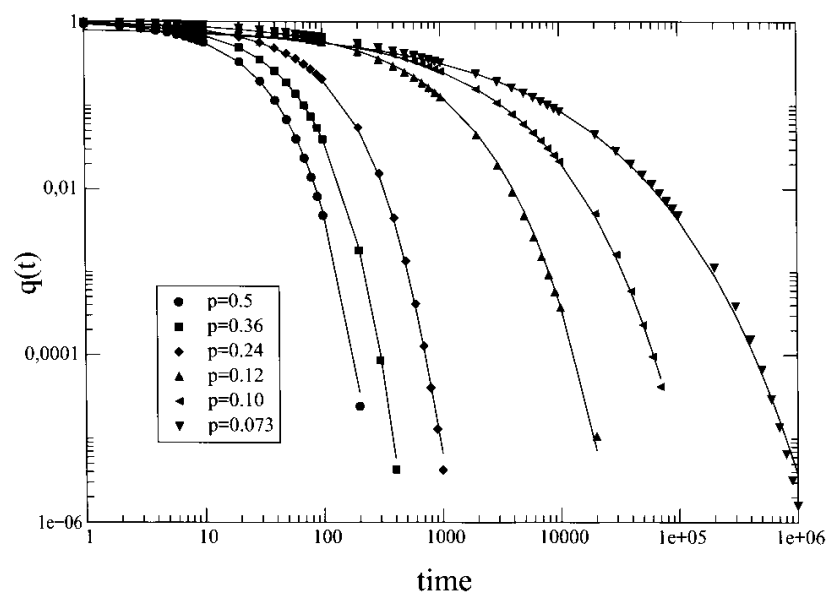

Figure 1. Decay of the autocorrelation function $q(t)$ on a log-log plot for different values of $p$, as listed in the inset. The solid lines correspond to stretched exponential fits, with $\beta(p)$ and $\tau(p)$ as indicated in Fig. (2). The error bars correspond to an estimate of the uncertainty of the points due to limited sampling.

In a more stringent test for the stretched exponential fit, Fig. 3 shows the same data as before but now in a $\log (-\log (q(\mathrm{t})))$ versus $\log (\mathrm{t})$ plot. In this kind of plots pure or stretched exponential functions appears as straight lines, with the slope giving the exponent $\beta$. As expected, all curves show deviations towards a pure exponential behavior for very short and very long times. At intermediate times, however, the stretched exponential behavior is clearly marked by the extended straight line regions. Also, both Figs. (2) and (3) show that as $p \rightarrow p_{c} \beta \rightarrow 1 / 3$ and $\tau \rightarrow \infty$.

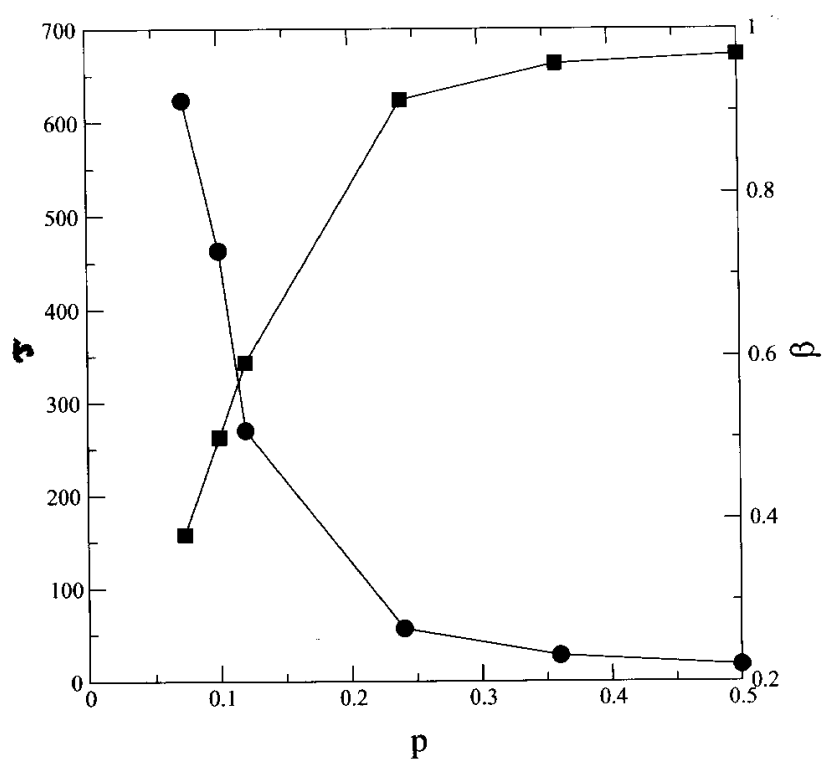

Figure 2. Relaxation time $\tau(p)$ (circles) and stretched exponential exponent $\beta(p)$ (squares) against $p$. As $p \rightarrow p_{c}, \tau$ diverges while $\beta(p)$ approaches $1 / 3$. 


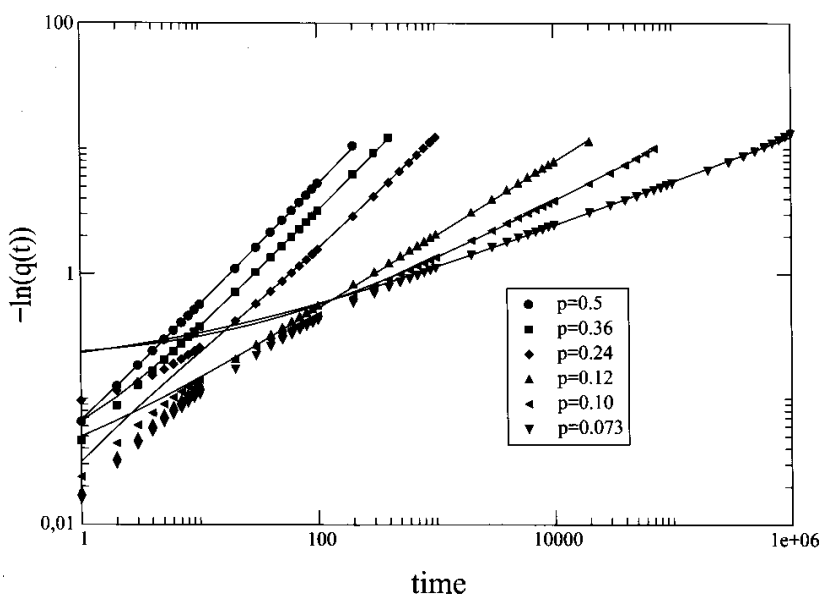

Figure 3. A more stringent test of the stretched exponential behavior of $q(t)$ is the $\log [-\log (q(t))]$ against $\log t$ plot. The different values of $p$ are listed in the inset. The solid lines correspond to a stretched exponential fit, with $\beta$ and $\tau$ as indicated in Fig.(2).

Finally, Fig. 4 shows the value of the local slope of $q(t)$ in the $\log (-\log (\mathrm{q}(\mathrm{t})))$ versus $\log (\mathrm{t})$ plot, that is, this figure presents the instantaneous value of the exponent $\beta$. The three regimes (exponential - stretched exponential - exponential) may clearly be spotted, with the minimum value for the instant value of $\beta$ approaching $1 / 3$ as the transition is approached.

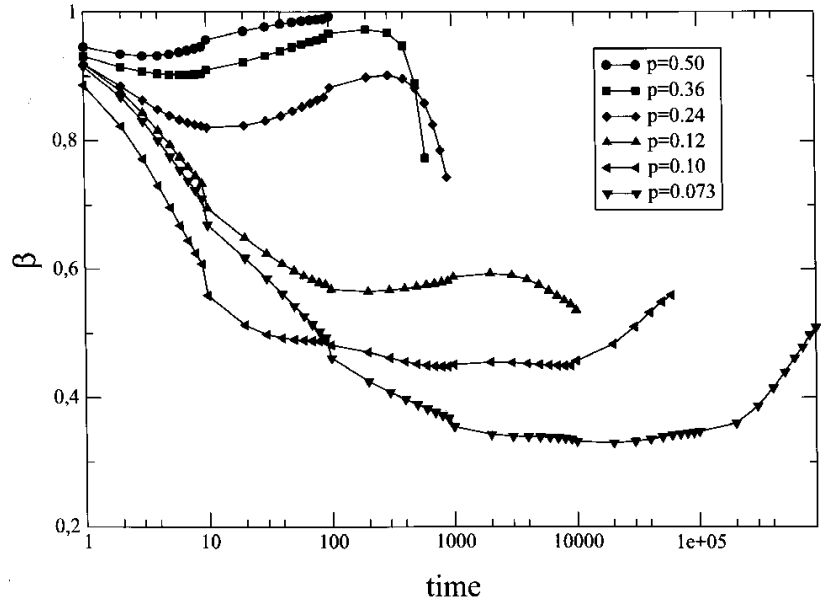

Figure 4. The instantaneous $\beta(t)$ versus $\log (t)$. For a perfect stretched exponential behavior the curves should be horizontal lines. The curves show that as we approach $p_{c}$ the plateaus get larger.

In summary, solutions of the master equation strongly confirm earlier numerical work on random walkers on the hypercube, but to much higher precision $[4-7]$.

\section{Conclusions}

The question may be raised as to whether the stretched exponential is the true limiting long time relaxation form in spin glasses. It has been stated that the long time relaxation should be dominated by large, compact, non-frustrated, isolated clusters of spins for temperatures below the Griffiths transition[10, 18, 19], but no numerical evidence has ever been found for the onset of this regime [18, 20, 21]. The probability of encountering large unfrustrated clusters in samples of the sizes studied numerically can be estimated and is microscopically small; thus any cluster- dominated regime would correspond to tiny values of $q(t)$ in huge samples, and so is unattainable in practice for numerical or experimental studies.

In systems with quenched-in disorder such as spin glasses, simulations show that there is some spatial heterogeneity of relaxation times, but that the relaxation of individual spins is generally strongly non-exponential $[11,12]$, in agreement with the fact that for each spin many different modes may be contributing to its relaxation, and different linear combinations of relaxation modes are established for different spins, depending on initial conditions and on the spatial delocalization of modes, as shown by the expression for the structure functions $f_{i}\left(\tau_{i}, \overrightarrow{S^{0}}\right)$ in Eq.(14). In this sense, local relaxation rates are expected to be heterogeneous and it may well happen that different sites exhibit the most rapid relaxation at different times. In spin-glass systems, where the probability of large unfrustrated regions is very small, the independent relaxing modes should not be expected to be localized, in contrast to systems where there are either independent relaxing clusters or independent hierarchical levels. Hence, in the presence of some quenched-in static inhomogeneity, heterogeneity can be expected to be mainly dynamic and the argument for an overall fractal phase space topology leading to the stretched exponential global relaxation is not affected.

In conclusion, using a master equation approach for random walks on the dilute hypercube, high precision results have been obtained compatible with the stretched exponential being the exact functional form for the decay in the limit of infinitely high dimension. Heterogeneities in local relaxation forms naturally appear when the autocorrelation functions are written in terms of the independent modes of relaxation of the density function defined on the hypercube. The stretched exponential appears as a consequence of fractality in a closed space, both for global and local relaxation functions: the sum over simple exponentials yielding a stretched exponential is then also valid for the local autocorrelation functions when the independent relaxation modes are not spatially localized. The resemblance between the dilute hypercube relaxation pattern and the relaxation actually observed in numerical studies of spin glasses or experiments on glasses above the freezing temperature strongly suggests that in physical systems the independent relaxation modes for the density function are not spatially localized and that the available region of the phase space may present 
a fractal structure in this temperature range. For sums such as those in Eqs.(13) and (15) to give rise to stretched exponentials, the weight of each different relaxation time component must have some characteristics such that, for example, a steepest-descent calculation of these sums yields a stretched exponential. As a stretched exponential appears for the hypercube near the percolation threshold, as indeed it is expected for random walkers over percolation clusters embedded on closed surfaces, we argue that the same type of topology should be present in the available region of Ising systems phase space near the glass transition, since these systems also present very similar patterns of relaxation in this temperature range.

\section{Acknowledgements}

This work has been partially supported by FrenchBrazilian Cooperation Program CAPES-COFECUB, and by Brazilian agencies FAPERGS and CNPq. We thank D. Stauffer for valuable suggestions.

\section{References}

[1] R. Kohlrausch, Pogg. Ann. Phys. Chem. 91, 179a (1854).

[2] M.D. Donsker and S.R.S. Varadhan, Commun. Pure Appl. Math. 32, 721 (1979), P. Grassberger and I. Procaccia, J. Chem. Phys. 77, 6281 (1982), J.C. Rasaiah, J. Zhu, J.B. Hubbard, and R.J. Rubin, J. Chem. Phys. 93, 5768 (1990).

[3] R.G. Palmer, D.L.Stein, E. Abrahams, and P.W. Anderson, Phys. Rev. Lett. 53, 958 (1984).

[4] I.A. Campbell, J. Phys. (France) Lett. 46, L1159 (1985).
[5] I. A. Campbell, J.M. Flesselles, R. Jullien, and R. Botet, J. Phys. C: Solid State Phys. 20, L47 (1987).

[6] I.A. Campbell, Europhys. Lett. 21, 959 (1993).

[7] N. Lemke and I. A. Campbell, Physica A 230, 554 (1996).

[8] R. M.C. de Almeida, N. Lemke and I. A. Campbell, European Physical Journal B, in press.

[9] J.C. Phillips, Rep. Prog. Phys. 59, 1133 (1996).

[10] M. Randeria, J.P. Sethna and R.G. Palmer, Phys. Rev. Lett. 54, 1321 (1985).

[11] T. Komori, K. Hukushima and H. Takayama, J. Phys. Soc. Japan 64, 4418 (1995).

[12] S.C. Glotzer, N. Jan, T. Lookman, A.B. MacIssac and P.H. Poole, Phys. Rev. E 57, 7350 (1998).

[13] R.B. Griffiths, Phys. Rev. Lett. 23, 17 (1969).

[14] S. Alexander and R. Orbach, J. Phys. Lett. 43, L625, (1982), Y. Gefen, A. Aharony and S. Alexander, Phys. Rev. Lett., 50, 77 (1983).

[15] P. Jund, R. Jullien and I.A. Campbell, to be published.

[16] P. Eördos and J. Spencer, Comput. Math. Appl. 5, 33 (1979); K.Weber, Elek. Inf. Verarb. Kybern. 22, 601 (1986).

[17] A. Bunde, S. Havlin, J. Klafter, G. Gräff, and A. Shehter, Phys. Rev. Lett. 78, 3338 (1998).

[18] A.J. Bray, J. Phys. A 22, LL81 (1989).

[19] F. Cesi, C. Maes and F. Martinelli, Comm. Math. Phys. 188, 135 (1997) and 189, 323 (1997).

[20] A.T. Ogielski, Phys. Rev. B 32, 7384 (1985).

[21] S. Jain, Physica A 218, 279 (1995). 